is continuous and as is readily seen single-valued. Thus the first term on the right taken along any closed path of the region is 0 . As to the second term, consider any closed path not including $c$. Then $F(z)$ and $F^{\prime}(z)=f(z)$ are both continuous throughout the enclosed region and hence the integral along the boundary of the region is $0 . *$ Finally let the path include $c$ and surround $c$ by a small circle. Then $\int F(z) d z$ extended in the usual way over the path and the circle will vanish. But the contribution that the circle yields is readily seen to be null; and thus $\int F(z) d z$ along any closed path whatever vanishes. Hence $\varphi(z)$ is analytic at $c$ too. Its derivative at $c$ is

$$
\lim _{z=c} \frac{\varphi(z)}{z-c}=\lim _{z=c} f(z)
$$

Hence $f(z)$ is continuous at $c$ and thus fulfills both conditions of the definition of an analytic function given in I, and the proof is complete. $\dagger$

HaRVARD UNIVERSITY, March 1896.

\title{
ON THE MOTION OF A HOMOGENEOUS SPHERE OR SPHERICAL SHELL ON AN INCLINED PLANE, TAKING INTO ACCOUNT THE ROTATION OF THE EARTH.
}

BY PROFESSOR ALEXANDRE S. CHESSIN.

The influence of the rotation of the earth on the motion of bodies on its surface has been the subject of most interesting experiments, of which those with falling bodies and those of Foucault with the pendulum and the gyroscop may be mentioned. We propose to give here another interesting illustration of that influence, namely, on the motion of a homogeneous sphere or spherical shell on a plane inclined to the horizon. We will identify the system of axes $(X, Y, Z)$ with the absolute, the system $(\Xi, r, Z)$ with the relative

* Cf. Goursat's proof of this theorem, Acta Math., vol. IV.; Harkness and Morley, Theory of Functions, p. 164.

+ Hölder has given in the Math. Annalen, vol. 20, 1882, a proof very similar to this. He integrates the given function $f(z)$ twice and shows that the second integral is analytic at $c$ too. Hence he infers that $f(z)$ is analytic at $c$. I had obtained the proof here given, however, before Hölder's proof was known to me. 
space, and first establish the differential equations of relative motion of a spherical shell in general.

Let $\xi_{i}, \eta_{i}, \zeta_{i}$ be the relative coördinates of a point $(i)$ of a moving body; $M$ its mass; $V_{\xi}, V_{\eta}, V_{\zeta}$ the components on the axes $\Xi, \Upsilon, Z$, of the principal vector of the real forces, $i . e$,

$$
V_{\xi}=\sum_{i} F_{i \xi} ; \quad V_{\eta}=\sum_{i} F_{i \eta} ; \quad V_{\zeta}=\sum_{i} F_{i \zeta} ;
$$

where $F_{i \xi}, F_{i \eta}, F_{i \zeta}$ are the components on the same axes of the real forces applied at a point $(i)$ of the body; in like manner let $V_{\xi}^{\prime}, V_{\eta}^{\prime}, V_{\xi}^{\prime}$ and $V_{\xi}^{\prime \prime}, V_{\eta}^{\prime \prime}, V_{\zeta}^{\prime \prime}$ be the components of the principal vectors of the fictitious forces of moving space and of compound accelerations, $i$. e.,

$$
\begin{gathered}
V_{\xi}^{\prime}=\sum_{i} m_{i} j_{c, \xi, i} \quad V_{\eta}^{\prime}=\sum_{i} m_{i} j_{c, \eta, i}, \quad V_{\zeta}^{\prime}=\sum_{i} m_{i} j_{c, \zeta, i} \\
V_{\xi}^{\prime \prime}=\sum_{i} m_{i} j_{s, \xi, i}, V_{\eta}^{\prime \prime}=\sum_{i} m_{i} j_{s, \eta, i}, \quad V_{\xi}^{\prime \prime}=\sum_{i} m_{i} j_{s, \zeta, i}
\end{gathered}
$$

where $m_{i}$ denotes the mass of a point $(i) ; j_{c, \xi, i,} j_{c, \eta, i}, j_{c, \zeta, i}$ denote the projections on the axes $\Xi, r, Z$, of the acceleration of the moving space at the point $(i)$ and $j_{s, \xi, i}, j_{s, \eta, i}, j_{s, \zeta, i}$ the projections on the same axes of the compound acceleration of that point; finally let $R_{\xi}, R_{\eta}, R_{\zeta}$, be the components of the principal vector of the reactions due to the conditions of constraint; then we can write down the following three differential equations of the motion of the centre of inertia (c) of the body :

$$
\begin{aligned}
& M \xi_{c}^{\prime \prime}=V_{\xi}-V_{\xi}^{\prime}+V_{\xi}^{\prime \prime}+R_{\xi} \\
& M \eta_{c}^{\prime \prime}=V_{\eta}-V_{\eta}^{\prime}+V_{\eta}^{\prime \prime}+R_{\eta} \\
& M \zeta_{c}^{\prime \prime}=V_{\zeta}-V_{\zeta}^{\prime}+V_{\zeta}^{\prime \prime}+R_{\zeta}
\end{aligned}
$$

The motion of the body about its centre of inertia will be defined by three differential equations, containing the projections of the moment of momentum of the absolute motion on a system of moving axes, having their origin at the centre of inertia of the body but otherwise independent of the same. Let $\mathfrak{X}, \mathfrak{V}, \exists$ denote the new axes; $m_{1}{ }^{(a)}, m_{2}{ }^{(a)}, m_{3}{ }^{(a)}$ be the components on these axes of the moment of absolute momentum about the centre of inertia of the body ; $M_{1}, M_{2}$, $M_{3}$ be the components on the same axes of the moment of the real forces; $\Lambda_{1}, \Lambda_{2}, \Lambda_{3}$ the components of the moment of the forces of reaction; finally let $\omega_{1}, \omega_{2}, \omega_{3}$ be the components of the angular velocity $(\omega)$ of the moving system 
( $\mathfrak{X}, \mathfrak{L}, \mathfrak{3})$. Then the three differential equations which we were to establish are:

$$
\begin{aligned}
& {\frac{d m_{1}}{d t}}^{(a)}=\omega_{3} m_{2}^{(a)}-\omega_{2} m_{3}^{(a)}+M_{1}+\Lambda_{1} \\
& \frac{d m_{2}^{(a)}}{d t}=\omega_{1} m_{3}^{(a)}-\omega_{3} m_{1}^{(a)}+M_{2}+\Lambda_{2} \\
& \frac{d m_{3}{ }^{(a)}}{d t}=\omega_{2} m_{1}^{(a)}-\omega_{1} m_{2}^{(a)}+M_{3}+\Lambda_{3}
\end{aligned}
$$

Let now the body be a homogenous spherical shell; let $H$ be its moment of inertia about a diameter ; $p^{(a)}, q^{(a)}, r^{(a)}$ the components on the axes $\mathfrak{X}, \mathfrak{Q}, \mathcal{J}$ of its absolute angular velocity ; then, as we know,

$$
m_{1}=H p^{(a)} ; m_{2}=H q^{(a)} ; m_{3}=H r^{(a)} .
$$

Further, let $p^{(r)}, q^{(r)}, r^{(r)}$ be the components on the same axes of the relative angular velocity of the spherical shell; $m_{1}{ }^{(r)}, m_{2}{ }^{(r)}, m_{3}{ }^{(r)}$ be the components of the moment of relative momentum about the center of inertia of the body; then

$$
\begin{gathered}
m_{1}{ }^{(a)}=H p^{(a)}=H\left(p^{(r)}+\omega_{1}\right)=m_{1}{ }^{(r)}+H \omega_{1} \\
m_{2}{ }^{(a)}=H q^{(a)}=H\left(q^{(r)}+\omega_{2}\right)=m_{2}^{(r)}+H \omega_{2} \\
m_{3}{ }^{(a)}=H r^{(a)}=H\left(r^{(r)}+\omega_{3}\right)=m_{3}{ }^{(r)}+H \omega_{3} \\
\frac{d m_{1}{ }^{(a)}}{d t}=\frac{d m_{1}^{(r)}}{d t}+H \frac{d \omega_{1}}{d t} \\
\frac{d m_{2}{ }^{(a)}}{d t}=\frac{d m_{2}{ }^{(r)}}{d t}+H \frac{d \omega_{2}}{d t} \\
\frac{d m_{3}{ }^{(a)}}{d t}=\frac{d m_{3}{ }^{(r)}}{d t}+H \frac{d \omega_{3}}{d t}
\end{gathered}
$$

Turning now to our problem, let $\mathrm{OZ}$ be the direction of the force of gravity; $\mathrm{XOY}$ the plane of the horizon; $\Xi 0 \mathrm{Y}$ the inclined plane forming an angle $\alpha$ with the horizon; $0 \Xi$ be the line of slope; $\beta$ the angle between the vertical plane $\mathrm{ZO} \Xi$ and the meridian of the point $\mathrm{O}$; the positive axis $0 Z$ be so taken as to make an acute angle with the direction of the force of gravity $(i . e$., with $\mathrm{O} Z$ ); finally let the axes $\mathfrak{X}, \mathfrak{L}, \mathfrak{Z}$ be taken parallel to the axes $\Xi, Y, Z$. Then we have

$$
\frac{d \omega_{1}}{d t}=\frac{d \omega_{2}}{d t}=\frac{d \omega_{3}}{d t}=0
$$


and therefore

$$
{\frac{d m_{1}}{d t}}^{(a)}={\frac{d m_{1}}{d t}}^{(r)} ;{\frac{d m_{2}}{d t}}^{(a)}=\frac{d m_{2}^{(r)}}{d t} \quad ; \quad{\frac{d m_{3}}{d t}}^{(a)}=\frac{d m_{3}^{(r)}}{d t}
$$

so that the equations (5) reduce to the following:

$$
\begin{aligned}
& \frac{d m_{1}^{(r)}}{d t}=\omega_{3} m_{2}^{(r)}-\omega_{2} m_{3}^{(r)}+M_{1}+\Lambda_{1} \\
& \frac{d m_{2}{ }^{(r)}}{d t}=\omega_{1} m_{3}^{(r)}-\omega_{3} m_{1}^{(r)}+M_{2}+\Lambda_{2} \\
& \frac{d m_{3}^{(r)}}{d t}=\omega_{2} m_{1}^{(r)}-\omega_{1} m_{2}^{(r)}+M_{3}+\Lambda_{3}
\end{aligned}
$$

The three equations (4) together with the three equations (11) determine the motion of the spherical shell relatively to the earth. The expressions for $\omega_{1}, \omega_{2}, \omega_{3}$ are readily found to be :

$$
\begin{aligned}
& \omega_{1}=\omega(\sin \lambda \sin \alpha+\cos \lambda \cos \alpha \cos \beta) \\
& \omega_{2}=\omega \cos \lambda \sin \beta \\
& \omega_{3}=\omega(\sin \lambda \cos \alpha-\cos \lambda \sin \alpha \cos \beta)
\end{aligned}
$$

$\omega$ being the angular velocity of the earth, and $\lambda$ being the latitude of the point $\mathrm{O}$.

In the following we will neglect terms of the order $\omega^{2}$ of smallness and higher, because the assumption that the force of gravity is constant within the whole space in which the motion of the spherical shell takes place in our example, is justified only if the results be required with an approximation up to terms of the order of $\omega$ and not higher. We then find :

$$
\begin{gathered}
\text { (13) } V_{\xi}=M g \sin \alpha ; \quad V_{\eta}=0 ; \quad V_{\zeta}=M g \cos \alpha \\
V_{\xi}^{\prime}=V_{\eta}^{\prime}=V_{\zeta}^{\prime}=0 \\
\text { (15) } V_{\xi}^{\prime \prime}=2 M \omega_{3} \eta_{c}^{\prime} ; V_{\eta}^{\prime \prime}=-2 M \omega_{3} \xi_{c}^{\prime} ; V_{\zeta}=-2 M\left(\omega_{1} \eta_{c}^{\prime}-\omega_{2} \xi_{c}^{\prime}\right)
\end{gathered}
$$

Let $\mathbf{R}$ be the reaction of the plane on which the sphere is moving; $F_{1}$ and $F_{2}$ the components of the force of friction between the sphere and the plane; then

$$
R_{\xi}=F_{1} ; \quad R_{\eta}=F_{2} ; \quad R_{\zeta}=\mathrm{R}
$$

and equations (4) become

$$
M \xi_{c}^{\prime \prime}=M g \sin \alpha+2 M \omega_{3} \eta^{\prime}+F_{1}
$$




$$
\begin{gathered}
M \eta_{c}^{\prime \prime}=-2 M \omega_{3} \xi_{c}^{\prime}+F_{2} \\
0=M g \cos \alpha-2 M\left(\omega_{1} \eta_{c}^{\prime}-\omega_{2} \xi_{c}^{\prime}\right)+\mathrm{R}
\end{gathered}
$$

If $a$ denote the radius of the exterior surface of the spherical shell we shall have

$$
\begin{gathered}
M_{1}=M_{2}=M_{3}=0 \\
\Lambda_{1}=-a F_{2} ; \quad \Lambda_{2}=a F_{1} ; \quad \Lambda_{3}=0
\end{gathered}
$$

and equations (11) become

$$
\begin{aligned}
& \frac{d p^{(r)}}{d t}-\omega_{3} q^{(r)}+\omega_{2} r^{(r)}=-\frac{a}{H} F_{2} \\
& \frac{d q^{(r)}}{d t}-\omega_{1} r^{(r)}+\omega_{3} p^{(r)}=\frac{a}{H} F_{1}
\end{aligned}
$$

$$
\frac{d r^{(r)}}{d t}-\omega_{2} p^{(r)}+\omega_{1} q^{(r)}=0
$$

To make our problem a determinate one, it is necessary to indicate the way in which the spherical shell moves on the plane. We will assume that it rolls on the plane without gliding. Then the relative velocity of the point of contact of spherical shell and plane is zero, which gives the two equations of condition

$$
\xi_{c}^{\prime}+a q^{(r)}=0 ; \quad \eta_{c}^{\prime}-a p^{(r)}=0
$$

If now we eliminate $F_{1}$ and $F_{2}$ from the seven equations $(17)_{1},(17)_{2},(20)_{1},(20)_{2},(20)_{3},(21)$, we obtain five equations between the five unknown quantities $\xi_{c}, \eta_{c}, p^{(r)}, q^{(r)}, r^{(r)}$, namely the three equations (20), and (21) and the following two :

$$
\begin{aligned}
& (22)_{1}\left(H+M \alpha^{2}\right) \frac{d g^{(r)}}{d t}=-M a g \sin \alpha-\left(H+2 M \alpha^{2}\right) \omega_{3} p^{(r)}+H \omega_{1} r^{(r)} \\
& (22)_{2} \quad\left(H+M \alpha^{2}\right) \frac{d p^{(r)}}{d t}=\left(H+2 M a^{2}\right) \omega_{3} q^{(r)}-H \omega_{2} r^{(r)}
\end{aligned}
$$

A rigorous integration of these equations, although very simple, is not necessary, since we neglect terms of the order higher than $\omega$. It is therefore preferable to integrate by successive differentiation, retaining every time only terms of the order not higher than $\omega$, which gives 


$$
\begin{aligned}
& p^{(r)}=p_{0}{ }^{(r)}+\frac{\left(H+2 M a^{2}\right) \omega_{3} q_{0}{ }^{(r)}-H \omega_{2} r_{0}^{(r)}}{H+M a^{2}} t \\
& -\frac{M a g \sin \alpha\left(H+2 M a^{2}\right)}{2\left(H+M a^{2}\right)^{2}} \omega_{3} t^{2}
\end{aligned}
$$

$(23)_{2} q^{(r)}=q_{0}^{(r)}-\frac{M a g \sin \alpha}{H+M a^{2}} t+\frac{H \omega_{1} r_{0}^{(r)}-\left(H+2 M a^{2}\right) \omega_{3} p_{0}^{(r)}}{H+M a^{2}} t$

$$
r^{(r)}=r_{0}^{(r)}+\left(\omega_{2} p_{0}^{(r)}-\omega_{1} q_{0}^{(r)}\right) t+\frac{M a g \sin \alpha}{2\left(H+M a^{2}\right)} \omega_{1} t^{2}
$$

where $p_{0}^{(r)}, q_{0}^{(r)}, r_{0}^{(r)}$ are the initial values of $p^{(r)}, q^{(r)}, r^{(r)}$. If we assume that the initial relative angular velocity of the spherical shell is zero, the above formulas reduce to the following :

$$
\begin{gathered}
p^{(r)}=-\frac{M a g \sin \alpha\left(H+2 M a^{2}\right)}{2\left(H+M a^{2}\right)^{2}} \omega_{3} t^{2} \\
q^{(r)}=-\frac{M a g \sin \alpha}{H+M a^{2}} t \\
r^{(r)}=\frac{M a g \sin \alpha}{2\left(H+M a^{2}\right)} \omega_{1} t^{2}
\end{gathered}
$$

The values given by (23) or (24) being substituted in (21) a new integration gives immediately

$$
\begin{gathered}
(25)_{1} \xi_{c}=\xi_{c 0}-a q_{0}{ }^{(r)} t+\frac{M a^{2} g \sin \alpha}{2\left(H+M a^{2}\right)} t^{2} \\
-\frac{a\left[H \omega_{1} r_{0}^{(r)}-\left(H+2 M a^{2}\right) \omega_{3} p_{0}{ }^{(r)}\right]^{2}}{2\left(H+M a^{2}\right)} t^{2} \\
(25)_{2} \quad \eta_{c}=\eta_{c 0}+a p_{0}{ }^{(r)} t+\frac{a\left[\left(H+2 M a^{2}\right) \omega_{3} q_{0}^{(r)}-H \omega_{2} r_{0}{ }^{(r)}\right]}{2\left(H+M a^{2}\right)} t^{2} \\
-\frac{M a^{2} g \sin \alpha\left(H+2 M a^{2}\right)}{6\left(H+M a^{2}\right)^{2}} \omega_{3} t^{3}
\end{gathered}
$$

We will give special attention to the case when $p_{0}{ }^{(r)}=q_{0}{ }^{(r)}$ $=r_{0}{ }^{(r)}=0$; if, besides, $\xi_{c 0}=\eta_{c 0}=0$ which can always be assumed by properly choosing the origin of the axes of coördinates, we obtain the following equations :

$$
\begin{gathered}
\xi_{c}=\frac{M \alpha^{2} g \sin \alpha}{2\left(H+M \alpha^{2}\right)} t^{2} \\
\eta_{c}=-\frac{M \alpha^{2} g \sin \alpha\left(H+2 M \alpha^{2}\right)}{6\left(H+M \alpha^{2}\right)^{2}} \omega_{3} t^{3}
\end{gathered}
$$

i. e., the centre of the spherical shell describes a semicubic parabola. 
In the case of a solid sphere $H=\frac{2}{5} M \alpha^{2}$ and

$$
\begin{gathered}
\xi_{c}=\frac{5}{14} g \sin \alpha t^{2} \\
\eta_{c}=-\frac{10}{49} g \sin \alpha \omega_{3} t^{3}
\end{gathered}
$$

and therefore the motion of the centre of a solid sphere does not depend on its mass or dimensions.

We see that the effect of the rotation of the earth on the motion of the centre of the sphere or spherical shell is to deviate it from the line of slope. This deviation may take place as well towards the east as towards the west of the meridian of the place according as $\omega_{3}$ is negative or positive, $i$. e., according as $\operatorname{tg} \lambda<\operatorname{tg} \alpha \cos \beta$ or $\operatorname{tg} \lambda>\operatorname{tg} \alpha \cos \beta$. If $\operatorname{tg} \lambda=\operatorname{tg} \alpha \cdot \cos \beta$ the rotation of the earth has no influence on the motion of the centre of the sphere or spherical shell.

These results may be interpreted geometrically as follows: Let $O P$ be drawn parallel to the axis of the earth (toward the south) and let $O Q$ be the projection of $O P$ on the plane $\Xi 0 \mathrm{Z}$. If then $\mu$ denote the angle of this projection with the horizon, we have

$$
\operatorname{tg} \lambda=\operatorname{tg} \mu \cos \beta
$$

Hence, if $\alpha<\mu$ the deviation of the sphere or spherical shell from the line of slope takes place toward the west; if $\alpha>\mu$ this deviation takes place toward the east; and if $\alpha=\mu$ the sphere or spherical shell will move along the line of slope.

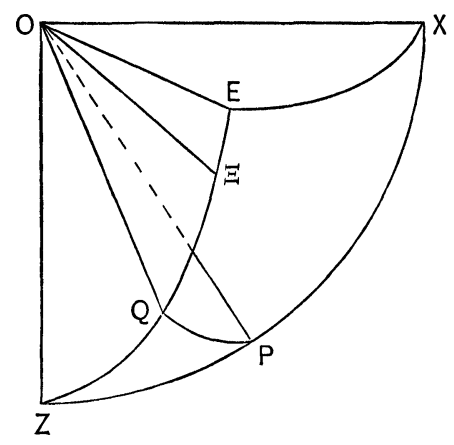

$$
\Varangle X O E=\beta \Varangle E O \Xi=a \Varangle E O Q=\mu \Varangle X O P=\lambda
$$


We have tacitly assumed that $-\frac{\pi}{2}<\beta<\frac{\pi}{2}$. However, the investigation of the different cases which may present themselves, although interesting, would carry us out of the limits of this paper. Let only one remark be made with respect to the variation of the angle $\beta$, namely, that the motion of the sphere or spherical shell depends on the orientation of the inclined plane and the deviation from the line of slope is maximum when $\beta=\pi$, minimum when $\beta=0$.

\section{NOTES.}

A REgular meeting of the American Mathematical Society was held in New York on Saturday afternoon, April 25, at three o'clock, Professor Mansfield Merriman in the chair. There were twenty-one members present. On the recommendation of the Council, the following persons, previously nominated, were elected to membership: Mr. F. M. McGAw, Bordentown Military Institute, Bordentown, N. J.; Mr. A. B. Chace, Valley Falls, R. I. ; Professor E. D. Roe, Oberlin College, Oberlin, Ohio. Four nominations for membership were received. The following papers were read :

(1) Dr. Emory McClintock: "On the most perfect forms of magic squares, with methods for their production."

(2) Professor Charlotte Angas Scott: "On the theory of the birational transformation of a plane curve."

THe committee appointed by the Council to arrange for holding a colloquium in connection with the summer meeting at Buffalo, announces two courses of six lectures each, one by Professor Maxime Bôcher on the subject of Linear Differential Equations and their Applications, the other by Professor James Pierpont on the Galois Theory of Equations. The meetings of the Colloquium will begin immediately after the adjournment of the Society and will continue through the week. A special circular giving detailed information in regard to the arrangements for the Colloquium will be issued by the Committee early in July.

A New list of officers and members of the Society has been compiled by the Secretary and has been published by the Society. In the same pamphlet are included the Constitution and By-Laws of the Society, an account of the last Annual Meeting, and the reports of the Secretary and the Treasurer for 1895. Extra copies can be obtained from the Secretary. 